\begin{tabular}{|c|c|}
\hline Title & Comparison of microtubules stabilized with the anticancer drugs cevipabulin and paclitaxel \\
\hline Author(s) & Nasrin, Syeda Rubaiya; Ishihara, T sukasa; Kabir, A rif Md Rashedul; Konagaya, A kihiko; Sada, Kazuki; Kakugo, A kira \\
\hline Citation & $\begin{array}{l}\text { Polymer journal, 52, 969-976 } \\
\text { https://doi .org/10.1038/s41428-020-0334-9 }\end{array}$ \\
\hline Issue Date & $2020-04-06$ \\
\hline Doc URL & http:/hdl.handle.net/2115/79421 \\
\hline Type & article (author version) \\
\hline Additional Information & There are other files related to this item in HUSCAP. Check the above URL. \\
\hline File Information & Polym. J.52_969-976.pdf \\
\hline
\end{tabular}

Instructions for use 


\section{Comparison of microtubules stabilized with the anticancer drugs cevipabulin and}

\section{paclitaxel}

Syeda Rubaiya Nasrin ${ }^{1}$, Tsukasa Ishihara ${ }^{2}$, Arif Md. Rashedul Kabir ${ }^{1}$, Akihiko Konagaya ${ }^{3}$, Kazuki Sada ${ }^{1,4}$, and Akira Kakugo*1,4

${ }^{1}$ Faculty of Science, Hokkaido University, Sapporo 060-0810, Hokkaido, Japan.

${ }^{2}$ Biomedical Research Institute, National Institute of Advanced Industrial Science and Technology (AIST), Tsukuba, Ibaraki, 305-8566, Japan.

${ }^{3}$ Department of Computational Intelligence and Systems Science, Tokyo Institute of Technology, Yokohama, 226-8502, Japan.

${ }^{4}$ Graduate School of Chemical Sciences and Engineering, Hokkaido University, Sapporo 060-0810, Hokkaido, Japan.

${ }^{*}$ Corresponding author

E-mail: kakugo@sci.hokudai.ac.jp

Tel/Fax: +81-11-706-3474 


\section{ABSTRACT}

Microtubules, one of the major components of the cytoskeleton, play important roles as pathways for neuronal transport of cellular traffic. Loss of structural stability of microtubules causes detrimental effects on neurons and may contribute to several neurodegenerative diseases, such as Alzheimer’s disease, amyotrophic lateral sclerosis, Huntington's disease, etc. The triazolopyrimidine class compound cevipabulin is a synthetic microtubule-stabilizing agent that has recently emerged as a drug for the treatment of Alzheimer's disease. However, the mechanism of microtubule stabilization by cevipabulin has not yet been revealed. Here, we explored the effect of cevipabulin on stabilizing microtubules polymerized from purified tubulins in vitro. We observed the effects of the concentration of microtubule-stabilizing drugs, incubation time, and modification of the cevipabulin structure on the stabilization of microtubules in comparison to those of the most commonly used anticancer drug, paclitaxel. This study will provide insight into the action of cevipabulin in the treatment of neurodegenerative diseases.

KEYWORDS: Microtubule, Dynamic instability, Microtubule-stabilizing agents, Cevipabulin, Paclitaxel 


\section{INTRODUCTION}

Microtubules perform roles in cellular processes, such as cell shape regulation, cell division, intracellular transportation, etc. ${ }^{1-4}$. The microtubule networks also provide pathways for intracellular cargo transport in neurons. These structures are dynamic cytoskeletal filaments consisting of $\alpha \beta$-tubulin heterodimers ${ }^{5}$. The heterodimers assemble in a head-to-tail fashion to form protofilaments that interact laterally to form the hollow cylindrical structure of microtubules ${ }^{6}$. The coexistence of the growing and shrinking states of microtubules is called dynamic instability ${ }^{7}$. In cells, microtubules are stabilized by microtubule-associated proteins, e.g., tau and MAP2 ${ }^{8,9}$. Any disruption in microtubule structure or function has been proposed to contribute to the key mechanism of neurodegenerative diseases, for example, Alzheimer's disease, amyotrophic lateral sclerosis, Huntington's disease, etc. ${ }^{10}$. Therefore, as a therapeutic strategy for neurodegenerative diseases, the stabilization of microtubules by halting the growth and shrinkage dynamics has been considered ${ }^{11,12}$. In vitro, the most widely used microtubulestabilizing agent is the plant-derived compound paclitaxel ${ }^{13}$. It has been used as a chemotherapeutic drug despite its severe disadvantages, such as insolubility in water ${ }^{14}$ and high toxicity ${ }^{15}$. Therefore, alternative synthetic compounds that can stabilize microtubules and thereby contribute to drug development for the treatment of 
neurodegenerative diseases are being explored ${ }^{12}$. One such synthetic compound is cevipabulin (5-chloro-6-[2,6-difluoro-4-[3-(methylamino)propoxy]phenyl]-N-[(2S)1,1,1-trifluoropropan-2-yl]-[1,2,4]triazolo[1,5-a]pyrimidin-7-amine) ${ }^{16-18}$. It is a small molecule with high water solubility and metabolic stability ${ }^{18}$. Cevipabulin has been highlighted in the treatment of neurological disorders ${ }^{12,19}$. Recently, it has been used to stabilize microtubules in vitro ${ }^{20,21}$. Several drug development studies have been performed using its derivatives ${ }^{22,23}$. However, a systematic comparison of the microtubule stabilization mechanisms of paclitaxel and cevipabulin remains elusive. In this study, we demonstrate the action of cevipabulin and one of its derivatives for stabilizing microtubules. We used (S)-6-(4-(3-butylamino(propoxy)-2,6-difluorophenyl)5-chloro-N-(1,1,1-trifluoro-propan-2-yl)-[1,2,4]triazolo[1,5-a]pyrimidin-7-amine as a derivative of cevipabulin (cevipabulin-R hereafter). We investigated the effect of the concentration of the drugs on the length and density of microtubules and the lifetime of the microtubules stabilized with those drugs by making a detailed comparison with the effect of paclitaxel.

\section{EXPERIMENTAL}

\section{Materials and methods}

\section{Chemicals}


All chemicals were purchased from Sigma-Aldrich, Inc. unless mentioned otherwise.

Cevipabulin was purchased from MedChemExpress (MCE).

\section{Preparation of the derivative of cevipabulin}

(S)-6-(4-(3-Butylamino(propoxy)-2,6-difluorophenyl)-5-chloro-N-(1,1,1-trifluoro-

propan-2-yl)-[1,2,4]triazolo[1,5-a]pyrimidin-7-amine, designated 'cevipabulin- $\mathrm{R}$ ' in the text hereafter, was synthesized as described below.

A mixture of 3-(butylamino)propan-1-ol (166 mg, $1.27 \mathrm{mmol}$ ) and sodium hydride (60\% oil suspension, $50.8 \mathrm{mg}, 1.27 \mathrm{mmol}$ ) in DMSO (3.0 mL) was stirred at ambient temperature for 1 h. (S)-5-Chloro-6-(2,4,6-trifluorophenyl)-N-(1,1,1-trifluoropropan-2yl)-[1,2,4]triazolo[1,5-a]pyrimidin-7-amine ${ }^{24}$ (200 $\mathrm{mg}, 0.506 \mathrm{mmol}$ ) was added to the mixture and stirred at $60{ }^{\circ} \mathrm{C}$ for $3 \mathrm{~h}$ (see SI S1.). The reaction mixture was cooled to ambient temperature, diluted with ethyl acetate, and washed with water and then brine. The organic layer was dried over sodium sulfate, filtered, and concentrated. The residue was purified by preparative HPLC to obtain (S)-6-(4-(3-butylamino(propoxy)-2,6difluorophenyl)-5-chloro-N-(1,1,1-trifluoro-propan-2-yl)-[1,2,4]triazolo[1,5a]pyrimidin-7-amine as a yellow oil (50 mg, 20\% yield). The 1H-NMR (400 MHz, $\mathrm{CDCl}_{3}$ ) spectrum was observed as follows: $\delta 0.94(\mathrm{t}, \mathrm{J}=7.2 \mathrm{~Hz}, 3 \mathrm{H}), 1.24-1.38(\mathrm{~m}, 4 \mathrm{H})$, 
$1.41(\mathrm{~d}, \mathrm{~J}=7.2 \mathrm{~Hz}, 3 \mathrm{H}), 1.50-1.57(\mathrm{~m}, 2 \mathrm{H}), 2.04-2.11(\mathrm{~m}, 2 \mathrm{H}), 2.70$ (t, J = $7.6 \mathrm{~Hz}$, 2H), 2.89 (t, J = 7.2 Hz, 2H), 4.13 (t, J = 6.4 Hz, 2H), 4.82—4.83 (m, $1 \mathrm{H}), 6.64-6.67$ (m, 2 H), 8.39 (s, 1H); ESI-MS m/z 507, 510 [M+H]+. Proton (1H) NMR spectra were recorded on a Varian Mercury plus $400 \mathrm{MHz}$ (see SI S2.). Chemical shifts are expressed in $\delta$ units, using tetramethylsilane as an internal standard (in the NMR description, $\mathrm{s}=$ singlet, $\mathrm{d}=$ doublet, $\mathrm{t}=$ triplet, and $\mathrm{m}=$ multiplet). Mass analysis was carried out on an Agilent 1200A with electrospray ionization.

\section{Molecular docking}

The binding interactions of the $\beta$-tubulin monomer with cevipabulin or cevipabulin- $\mathrm{R}$

were visualized using the molecular modeling system UCSF Chimera ${ }^{25}$. The docking was performed based on a simple scoring function and rapid gradient-optimization conformational search using AutoDock/Vina software ${ }^{26}$. The receptor used for the study was the $\beta$-tubulin monomer (PDB ID: 1TUB), and the ligands were the (a) cevipabulin and (b) cevipabulin-R molecules.

\section{Purification, labeling, and polymerization of tubulin}

Tubulin was purified from fresh porcine brain using high-concentration PIPES buffer (1 M PIPES, 20 mM EGTA, 10 mM MgCl $2 ;$ pH 6.8) ${ }^{27}$. ATTO 550 fluorescent dye-labeled 
tubulin was obtained following a standard protocol ${ }^{28}$. Microtubules were polymerized from $56 \mu \mathrm{M}$ tubulin (20\% ATTO 550-labeled tubulin and 80\% nonlabeled tubulin) in BRB80 (80 mM PIPES, 1 mM MgCl 21 mM ethylene glycol-bis( $\beta$-aminoethyl ether)$\mathrm{N}, \mathrm{N}, \mathrm{N}^{\prime}, \mathrm{N}^{\prime}$-tetraacetic acid (EGTA); $\mathrm{pH}$ 6.8) in the presence of $5 \mathrm{mM}$ guanosine 5'triphosphate (GTP), $20 \mathrm{mM} \mathrm{MgCl}_{2}$, and 25\% dimethyl sulfoxide incubated at $37{ }^{\circ} \mathrm{C}$ for 60 minutes.

\section{Fluorescence microscopy observation}

Microtubule samples were illuminated with a $100 \mathrm{~W}$ mercury lamp and observed through an epifluorescence microscope (Eclipse Ti; Nikon Instruments Inc.) equipped with an oilcoupled Plan Apo 60×1.40 objective (Nikon Instruments Inc.). A filter block with UVcutoff specifications (TRITC: EX540/25, DM565, BA606/55, Nikon Instruments Inc.) was used in the optical path of the microscope to eliminate the UV radiation and minimize the harmful effect of UV radiation on samples during observation. Images were captured using a cooled CMOS (complementary metal-oxide-semiconductor) camera (Neo CMOS; Andor, Oxford Instruments Company) connected to a PC.

\section{Microtubule density measurement}


The number of microtubules per frame was counted. The area of each frame was $2560 \times 2160$ pixels $^{2}$ or $280 \times 237 \mu^{2}$. The number of microtubules per unit area was defined as the density of the microtubules.

\section{Data analysis}

NIS-Elements BR software (Nikon Instruments Inc.) and Fiji 1.52J software (National Institutes of Health, USA) were used to analyze the fluorescence microscopy images. Molecular structures of the compounds used were drawn using Biovia Draw 2019 software. For plotting graphs, GraphPad Prism 8 software was used.

\section{RESULTS AND DISCUSSION}

In Fig. 1, the molecular structures of cevipabulin (Fig. 1 (a)) and its derivative, cevipabulin-R, (Fig. 1 (b)), used in our study are provided. These molecules are smaller and have simpler structures than the most widely used microtubule-stabilizing agent, paclitaxel (Fig. 1 (c)). Dynamic microtubule polymers are assembled from heterodimers consisting of $\alpha$ - and $\beta$-tubulin. Microtubule-stabilizing drugs that target the $\beta$-tubulin monomer facilitate the lateral or longitudinal tubulin-tubulin interaction in microtubules and stabilize microtubules against depolymerization (Fig. S1). Cevipabulin has previously been shown to bind to both the taxoid site and the vinca alkaloid domain of $\beta$ - 
tubulin of microtubules ${ }^{20}$. In this study, we used a modified cevipabulin structure, with a longer alkyl side chain attached to the terminal $\mathrm{N}$-atom of the molecule. First, we carried out molecular docking on the $\beta$-tubulin-cevipabulin and $\beta$-tubulin-cevipabulin-R pairs based on a simple scoring function ${ }^{26}$. In this task, $\beta$-tubulin was assigned as the receptor and the cevipabulin or cevipabulin-R molecule was assigned as the ligand. We performed a rapid gradient-optimization conformational search to obtain the binding affinity between the receptor-ligand species ${ }^{26}$. The superimposed structures gave the 3D structures of the $\beta$-tubulin-drug complexes. In Fig. 2, the three most energetically stable structures of the complexes are provided. The output from the 10 best modes of the complexes from Chimera Protein-Ligand Docking is given in Table 1. The results showed that the binding affinity of cevipabulin to $\beta$-tubulin $(-7.9 \mathrm{kcal} / \mathrm{mol})$ was slightly higher than that of cevipabulin- $\mathrm{R}(-7.7 \mathrm{kcal} / \mathrm{mol})$. The best docked configuration of the paclitaxel molecule complexed within the active site of 1TUB was reported to be $-11.46 \mathrm{kcal} / \mathrm{mol}$ 29.

Table 1. Chimera Protein-Ligand Docking results (for GDP tetrahedral and 0 charges assigned). The energetically most favorable complexes are marked with asterisks.

a) Receptor: TUB ( $\beta$-chain); Ligand: cevipabulin

\begin{tabular}{|c|c|}
\hline Mode & Distance from best mode $(\AA)$ \\
\hline
\end{tabular}




\begin{tabular}{|c|c|c|c|c|c|c|}
\hline & $\begin{array}{c}\text { Binding } \\
\text { affinity } \\
\text { (kcal/mol) }\end{array}$ & $\begin{array}{c}\underline{\mathrm{RMSD}} \\
\text { lower bound }\end{array}$ & $\begin{array}{c}\underline{\mathrm{RMSD}} \\
\text { upper bound }\end{array}$ & $\frac{\text { H-bonds }}{\underline{\text { all }}}$ & $\frac{\underline{\text { H-bond }}}{\underline{\text { ligand }}}$ & $\frac{\underline{\text { H-bond }}}{\underline{\text { receptor }}}$ \\
\hline $1^{*}$ & -7.9 & 0.000 & 0.000 & 2 & 2 & 2 \\
\hline 2 & -7.3 & 11.589 & 13.832 & 1 & 1 & 1 \\
\hline $3^{*}$ & -7.3 & 2.094 & 2.910 & 1 & 1 & 1 \\
\hline 4 & -7.1 & 11.396 & 13.682 & 0 & 0 & 0 \\
\hline 5 & -7.1 & 11.442 & 12.956 & 1 & 1 & 1 \\
\hline 6 & -7.0 & 11.888 & 13.951 & 1 & 1 & 1 \\
\hline 7 & -7.0 & 11.359 & 12.718 & 0 & 0 & 0 \\
\hline $8 *$ & -6.9 & 1.856 & 2.249 & 0 & 0 & 0 \\
\hline 9 & -6.8 & 27.941 & 29.652 & 1 & 1 & 1 \\
\hline 10 & -6.7 & 12.908 & 14.576 & 2 & 2 & 2 \\
\hline \multicolumn{7}{|c|}{ b) Receptor: TUB ( $\beta$-chain); Ligand: cevipabulin-R } \\
\hline $11 *$ & -7.7 & 0.000 & 0.000 & 0 & 0 & 0 \\
\hline 12 & -7.6 & 32.063 & 35.311 & 1 & 1 & 1 \\
\hline 13 & -7.5 & 3.518 & 8.197 & 2 & 2 & 2 \\
\hline $14^{*}$ & -7.5 & 2.548 & 3.702 & 2 & 2 & 2 \\
\hline 15 & -7.0 & 3.678 & 4.847 & 0 & 0 & 0 \\
\hline 16 & -7.0 & 32.036 & 35.218 & 2 & 2 & 2 \\
\hline 17 & -6.9 & 27.909 & 31.774 & 1 & 1 & 1 \\
\hline $18 *$ & -6.8 & 3.189 & 4.375 & 1 & 1 & 1 \\
\hline 19 & -6.8 & 33.867 & 36.165 & 0 & 0 & 0 \\
\hline 20 & -6.7 & 3.631 & 8.207 & 0 & 0 & 0 \\
\hline
\end{tabular}

We obtained the 10 most energetically stable conformers of the tubulin-cevipabulin and tubulin-cevipabulin-R complexes from the docking. The RMSD (root mean square deviation of atomic positions) of a particular mode refers to the distance of atoms of the same molecule in the best mode. It depends on the binding interaction energy between the receptor and the ligand of the complex. Lower RMSD values of complexes correspond 
to more stable structures of the complexes. For an acceptable docking pose quality, RMSD values should be lower than $3 \AA^{30}$. Therefore, we obtained the three best modes of the complexes for each of the $\beta$-tubulin-cevipabulin and $\beta$-tubulin-cevipabulin-R pairs based on the docking scores and lowest RMSD values ${ }^{31}$. The three most favorable structures are provided in Fig. 2. We observed that in all six structures, the binding between the $\beta$-tubulin monomer and both drug molecules takes place at the taxoid binding site $^{32}$. However, complexes showing interactions of drug molecules at the alkaloid domain of the $\beta$-tubulin monomer were also observed (Figs. S2 and S3).

At present, there is no evidence that shows whether cevipabulin-R can be utilized in stabilizing microtubules against depolymerization. We polymerized tubulins to microtubules as described in the Experimental section and checked the effectiveness of cevipabulin and cevipabulin- $\mathrm{R}$ in microtubule stabilization. To explore the action of cevipabulin and cevipabulin-R on microtubules, we investigated the effect of different drug concentrations on microtubule stabilization.

Tubulin was polymerized into microtubules in the presence of GTP for $1 \mathrm{~h}$. After that, the microtubules were diluted in a 1:9 volume ratio with different buffers containing 0.1 $\mu \mathrm{M}$ to $100.0 \mu \mathrm{M}$ cevipabulin, cevipabulin- $\mathrm{R}$ or paclitaxel. The fluorescence microscopy images of the microtubules stabilized using $0.1,5.0,50.0$, and $100.0 \mu \mathrm{M}$ cevipabulin, 
cevipabulin-R, and paclitaxel are shown in Fig. 3 (a), (b) and (c), respectively. In the presence of $0.1 \mu \mathrm{M}$ paclitaxel, few microtubule filaments could be observed. However, no microtubule filaments were stabilized using $0.1 \mu \mathrm{M}$ cevipabulin or cevipabulin-R. We observed microtubules in the presence of a $5.0 \mu \mathrm{M}$ concentration of all three drugs. Therefore, it was established that both cevipabulin and cevipabulin-R are able to stabilize microtubules. The average initial lengths of the microtubules stabilized by $5.0 \mu \mathrm{M}$ cevipabulin, cevipabulin-R and paclitaxel were $10.97 \pm 6.50 \mu \mathrm{m}, \mathrm{n}=137,11.89 \pm 7.67 \mu \mathrm{m}$, $\mathrm{n}=244$, and $12.31 \pm 7.81 \mu \mathrm{m}, \mathrm{n}=279$, respectively. The similar values of the mean microtubule lengths indicate that equimolar tubulin and drug content (at $5.0 \mu \mathrm{M}$, tubulin:drug $=1.2: 1.0$ ) is capable of stabilizing the microtubules against depolymerization. At a tubulin-to-drug molar concentration ratio of 1.0:8.0, a $50.0 \mu \mathrm{M}$ concentration, extended microtubules with comparable lengths were obtained (16.11 $\pm 10.51 \mu \mathrm{m}, \mathrm{n}=129$ with $50.0 \mu \mathrm{M}$ cevipabulin, $13.83 \pm 7.75 \mu \mathrm{m}, \mathrm{n}=156$ with $50.0 \mu \mathrm{M}$ cevipabulin-R, and $12.31 \pm 9.07 \mu \mathrm{m}, \mathrm{n}=205$ with $50.0 \mu \mathrm{M}$ paclitaxel). We observed microtubules stabilization using up to a $100.0 \mu \mathrm{M}$ concentration of the drugs. In this high concentration range, we found large tubulin aggregates along with stable microtubules.

The lengths and densities of the microtubules incubated across the range of drug concentrations are given in Fig. 4 (a) and Fig. 4(b), respectively. We observed that high 
concentrations of the microtubule-stabilizing agents cevipabulin, cevipabulin- $\mathrm{R}$, and paclitaxel could ensure microtubule stabilization. The promotion of tubulin polymerization in the presence of paclitaxel has long been established ${ }^{33}$. Our study provides a similar finding for a molecule from another class of microtubule-stabilizing drugs, cevipabulin.

We compared the mechanical properties of microtubules stabilized by cevipabulin, cevipabulin-R, or paclitaxel. The microtubules stabilized by cevipabulin have been reported to be more flexible than those stabilized by paclitaxel. As a measure of rigidity, we estimated the persistence length of microtubules according to the literature ${ }^{21,34}$. We used the following equation:

$$
R^{2}=2 L_{p}^{2}\left[\frac{L}{L_{p}}-1+e^{\frac{L}{L p}}\right]
$$

where $\mathrm{R}$ and $\mathrm{L}$ are the mean squared end-to-end length and the contour length of the microtubule, respectively. The derived parameter $L_{p}$ gives the persistence length of the microtubule. The persistence lengths of the microtubules stabilized by cevipabulin, cevipabulin-R and paclitaxel are given in Fig. 5. The $\mathrm{L}_{\mathrm{p}}$ values of cevipabulin-stabilized $(60.99 \pm 8.78 \mu \mathrm{m}, \mathrm{n}=90)$ and paclitaxel-stabilized microtubules $(83.47 \pm 16.52 \mu \mathrm{m}, \mathrm{n}$ $=90$ ) are consistent with those in a previous report ${ }^{21}$. In this study, we obtained an Lp for cevipabulin-R-stabilized microtubules $(58.16 \pm 7.89 \mu \mathrm{m}, \mathrm{n}=90)$ that was almost equal to 
that for the cevipabulin-stabilized microtubules. Such a result confirms the similar effect of cevipabulin and cevipabulin-R on the rigidity of microtubules, which is expected due to the similarity in the structures of the drug molecules.

In Fig. 6(a), we show the effect on the lifetime of the microtubules in buffers containing different microtubule-stabilizing agents of the same concentration $(50 \mu \mathrm{M})$. We measured the lengths of the microtubules at $0,6,12$, and $24 \mathrm{~h}$ of incubation and plotted the mean microtubule length as a function of the incubation time (Fig. 6(a)). The mean lengths of the microtubules with the numbers of microtubules analyzed at different incubation times are given in Table 2 .

Table 2. The mean microtubule lengths of microtubules stabilized with different microtubule-stabilizing drugs after incubation for $0,6,12$, or $24 \mathrm{~h}$. Here, $\mathrm{n}=$ number of microtubules analyzed, and SD= standard deviation. The results are shown in Fig. 6(a).

\begin{tabular}{|c|c|c|c|c|c|c|}
\hline \multirow{2}{*}{$\begin{array}{c}\text { 导 } \\
\text { E }\end{array}$} & \multicolumn{2}{|c|}{$\begin{array}{c}\text { Cevipabulin } \\
\text { stabilized }\end{array}$} & $\begin{array}{c}\text { Mean length } \\
\pm \mathrm{SD}(\mu \mathrm{m})\end{array}$ & $\mathrm{n}$ & $\begin{array}{c}\text { Cevipabulin-R } \\
\text { stabilized } \\
\pm \mathrm{SD}(\mu \mathrm{m})\end{array}$ & \multicolumn{2}{c|}{$\begin{array}{c}\text { Paclitaxel } \\
\text { stabilized }\end{array}$} \\
\cline { 2 - 7 } & $10.18 \pm 5.47$ & 174 & $10.79 \pm 6.04$ & 129 & $\begin{array}{c}\text { Mean length } \\
\pm \mathrm{SD}(\mu \mathrm{m})\end{array}$ & $\mathrm{n}$ \\
\hline 0 & $8.12 \pm 3.85$ & 216 & $8.33 \pm 4.43$ & 90 & $14.24 \pm 8.65$ & 136 \\
\hline 6 & $7.70 \pm 2.95$ & 100 & $7.90 \pm 3.74$ & 94 & $18.62 \pm 10.14$ & 102 \\
\hline 12 & $6.47 \pm 2.85$ & 156 & $8.41 \pm 3.55$ & 133 & $20.11 \pm 11.63$ & 119 \\
\hline 24 & & & & & & \\
\hline
\end{tabular}


We found that the microtubules stabilized using paclitaxel became significantly longer with incubation time. Such a phenomenon indicates the favored polymerization of microtubules in the presence of paclitaxel. The microtubule lengths were not significantly altered when stabilized by cevipabulin or its derivative, indicating that cevipabulin can stabilize microtubules for an extended time. We also found that the densities of paclitaxelstabilized microtubules decreased with time, as shown in Fig. 6(b). Annealing of microtubules with prolonged incubation in paclitaxel-containing buffers may have resulted in such observations ${ }^{35,36}$. Microtubules incubated with cevipabulin or its derivative did not exhibit a similar increase in length and decrease in the densities of the stabilized microtubules with time. A rather slight shortening of the cevipabulin- or cevipabulin-R-stabilized microtubules was observed with time. Therefore, we speculate that cevipabulin does not promote tubulin elongation or annealing of microtubules. In some instances, the microtubule density with cevipabulin- $\mathrm{R}$ became double that with cevipabulin. Furthermore, we noticed that there were variations in the density and length of microtubules that corresponded with the variable concentration of cevipabulin-R, which did not follow a regular trend. Therefore, the reason behind such differences in the densities of microtubules stabilized by cevipabulin or cevipabulin-R is not currently clear and requires a detailed investigation in the future. 


\section{CONCLUSION}

The study we present here provides a systematic investigation of the difference in the microtubule stabilization mechanisms of cevipabulin and paclitaxel. Modification of the structure of cevipabulin did not significantly alter its interaction with microtubules, suggesting that the modified drug is also efficient in the stabilization of microtubules. Furthermore, our study proves that the action of microtubule stabilization by paclitaxel varies from that by cevipabulin or its derivative. Such diversity in the microtubule stabilization mechanism may present extended opportunities for the application of microtubule-modulating agents in the treatment of neurodegenerative diseases ${ }^{12,19}$ as well as in the construction of biofunctional nanodevices ${ }^{37,38}$. 


\section{ACKNOWLEDGMENTS}

This work was financially supported by the Robot Technology Research and

Development Project from New Energy and Industrial Technology Development Organization (NEDO), Japan, Grant-in-Aid for Scientific Research on Innovative Areas

“Molecular Engine” (JP18H05423 and JP18H03673) and Grant-in-Aid for Young Scientists (A) to A.K. from the Japan Society for the Promotion of Science (JSPS).

\section{REFERENCES}

1. Howard, J. Mechanics of Motor Proteins and the Cytoskeleton. (Sinauer Associates, Inc., Sunderland, Massachusetts, 2001).

2. Doodhi, H., Prota, A. E., Rodriguez-Garcia, R., Xiao, H., Custar, D. W., Bargsten, K., Katrukha, E. A., Hilbert, M., Hua, S., Jiang, K., Grigoriev, I., Yang, C.-P. H., Cox, D., Horwitz, S. B., Kapitein, L. C., Akhmanova, A. \& Steinmetz, M. O. Termination of protofilament elongation by eribulin induces lattice defects that promote microtubule catastrophes. Curr. Biol. 26, 1713-1721 (2016).

3. Scholey, J. M., Brust-Mascher, I. \& Mogilner, A. Cell division. Nature 422, 746752 (2003). 
4. Ross, J. L., Ali, M. Y. \& Warshaw, D. M. Cargo transport: molecular motors navigate a complex cytoskeleton. Curr. Opin. Cell Biol. 20, 41-47 (2008).

5. Alberts, P. B., Johnson, A., Lewis, J., Raff, M., Roberts, K. \& Walter P. Molecular biology of cell. (Garland Science, New York, 2008).

6. Amos, L. A. \& Baker, T. S. The three-dimensional structure of tubulin protofilaments. Nature 279, 607-612 (1979).

7. Mitchison, T., Kirschner, M. \& Mitchison T, K. M. Dynamic instability of microtubule growth. Nature 312, 237-42 (1984).

8. Drechsel, D. N., Hyman, A. A., Cobb, M. H. \& Kirschner, M. W. Modulation of the dynamic instability of tubulin assembly by the microtubule-associated protein tau. Mol. Biol. Cell 3, 1141-1154 (1992).

9. Itoh, T. J. \& Hotani, H. Microtubule-Stabilizing Activity of MicrotubuleAssociated Proteins (MAPs) Is Due to Increase in Frequency of Rescue in Dynamic Instability: Shortening Length Decreases with Binding of MAPs onto Microtubules. Cell Struct. Funct. 19, 279-290 (1994).

10. Dubey, J., Ratnakaran, N. \& Koushika, S. P. Neurodegeneration and microtubule 
dynamics: Death by a thousand cuts. Front. Cell. Neurosci. 9, 343 (2015).

11. Giannakakou, P., Nakano, M., Nicolaou, K. C., O’Brate, A., Yu, J., Blagosklonny, M. V., Greber, U. F. \& Fojo, T. Enhanced microtubule-dependent trafficking and p53 nuclear accumulation by suppression of microtubule dynamics. Proc. Natl. Acad. Sci. U. S. A. 99, 10855-10860 (2002).

12. Brunden, K. R., Trojanowski, J. Q., Smith, A. B., Lee, V. M. Y. \& Ballatore, C. Microtubule-stabilizing agents as potential therapeutics for neurodegenerative disease. Bioorganic Med. Chem. 22, 5040-5049 (2015).

13. Schiff, P. B. \& Horwitz, S. B. Taxol stabilizes microtubules in mouse fibroblast cells. Proc. Natl. Acad. Sci. U. S. A. 77, 1561-1565 (1980).

14. Dordunoo, S. K. \& Burt, H. M. Solubility and stability of taxol: Effects of buffers and cyclodextrins. Int. J. Pharm. 133, 191-201 (1996).

15. Nicoletti, M. I., Colombo, T., Rossi, C., Monardo, C., Stura, S., Zucchetti, M., Riva, A., Morazzoni, P., Donati, M. B., Bombardelli, E., D’Incalci, M. \& Giavazzi, R. IDN5109, a taxane with oral bioavailability and potent antitumor activity. Cancer Res. 60, 842-846 (2000). 
16. Sáez-Calvo, G., Sharma, A., Balaguer, F. de A., Barasoain, I., Rodríguez-

Salarichs, J., Olieric, N., Muñoz-Hernández, H., Berbís, M. Á., Wendeborn, S., Peñalva, M. A., Matesanz, R., Canales, Á., Prota, A. E., Jímenez-Barbero, J., Andreu, J. M., Lamberth, C., Steinmetz, M. O. \& Díaz, J. F. Triazolopyrimidines are microtubule-stabilizing agents that bind the vinca inhibitor site of tubulin.

Cell Chem. Biol. 24, 737-750 (2017).

17. Beyer, C. F., Zhang, N., Hernandez, R., Vitale, D., Lucas, J., Nguyen, T., Discafani, C., Ayral-Kaloustian, S. \& Gibbons, J. J. TTI-237: A novel microtubule-active compound with in vivo antitumor activity. Cancer Res. 68, 2292-2300 (2008).

18. Zhang, N., Ayral-Kaloustian, S., Nguyen, T., Afragola, J., Hernandez, R., Lucas, J., Gibbons, J. \& Beyer, C. Synthesis and sar of [1,2,4]triazolo[1,5-a]pyrimidines, a class of anticancer agents with a unique mechanism of tubulin inhibition. $J$. Med. Chem. 50, 319-327 (2007).

19. Ballatore, C., Brunden, K. R., Trojanowski, J. Q., Lee, V. M. Y. \& Smith, A. B. Non-naturally occurring small molecule microtubule-stabilizing agents: a potential tactic for CNS-directed therapies. ACS Chem. Neurosci. 8, 5-7 (2017). 
20. Beyer, C. F., Zhang, N., Hernandez, R., Vitale, D., Nguyen, T., Ayral-

Kaloustian, S. \& Gibbons, J. J. The microtubule-active antitumor compound TTI-

237 has both paclitaxel-like and vincristine-like properties. Cancer Chemother.

Pharmacol. 64, 681-689 (2009).

21. Nasrin, S. R., Kabir, A. M. R., Konagaya, A., Ishihara, T., Sada, K. \& Kakugo,

A. Stabilization of microtubules by cevipabulin. Biochem. Biophys. Res.

Commun. 516, 760-764 (2019).

22. Zhang, N., Ayral-Kaloustian, S., Nguyen, T., Hernandez, R., Lucas, J., Discafani,

C. \& Beyer, C. Synthesis and SAR of 6-chloro-4-fluoroalkylamino-2-heteroaryl-

5-(substituted)phenylpyrimidines as anti-cancer agents. Bioorganic Med. Chem.

17, 111-118 (2009).

23. Zhang, N., Ayral-Kaloustian, S., Nguyen, T., Hernandez, R. \& Beyer, C. 2Cyanoaminopyrimidines as a class of antitumor agents that promote tubulin polymerization. Bioorganic Med. Chem. Lett. 17, 3003-3005 (2007).

24. Lou, K., Yao, Y., Hoye, A. T., James, M. J., Cornec, A. S., Hyde, E., Gay, B., Lee, V. M. Y., Trojanowski, J. Q., Smith, A. B., Brunden, K. R. \& Ballatore, C. Brain-penetrant, orally bioavailable microtubule-stabilizing small molecules are 
potential candidate therapeutics for Alzheimer's disease and related tauopathies.

J. Med. Chem. 57, 6116-6127 (2014).

25. UCSF Chimera Home Page. at <http://www.cgl.ucsf.edu/chimera/>

26. Olson, Arthur J., O. T. AutoDock Vina: improving the speed and accuracy of docking with a new scoring function, efficient optimization and multithreading. NIH Pubic Access 31, 455-461 (2011).

27. Castoldi, M. \& Popov, A. V. Purification of brain tubulin through two cycles of polymerization-depolymerization in a high-molarity buffer. Protein Expr. Purif. 32, 83-88 (2003).

28. Peloquin, J., Komarova, Y. \& Borisy, G. Conjugation of fluorophores to tubulin. Nat. Methods 2, 299-303 (2005).

29. Yadava, U., Gupta, H. \& Roychoudhury, M. Stabilization of microtubules by taxane diterpenoids: insight from docking and MD simulations. J. Biol. Phys. 41, 117-133 (2015).

30. Li, Y., Han, L., Liu, Z. \& Wang, R. Comparative assessment of scoring functions on an updated benchmark: 2. evaluation methods and general results. J. Chem. 
Inf. Model. 54, 1717-1736 (2014).

31. Srivastava, H. K., Chourasia, M., Kumar, D. \& Sastry, G. N. Comparison of computational methods to model DNA minor groove binders. J. Chem. Inf. Model. 51, 558-571 (2011).

32. Rao, S., He, L., Chakravarty, S., Ojima, I., Orr, G. A. \& Horwitz, S. B. Characterization of the Taxol binding site on the microtubule. Identification of $\operatorname{Arg} 282$ in $\beta$-tubulin as the site of photoincorporation of a 7-benzophenone analogue of Taxol. J. Biol. Chem. 274, 37990-37994 (1999).

33. Kumar, N. Taxol-induced polymerization of purified tubulin. Mechanism of action. J. Biol. Chem. 256, 10435-10441 (1981).

34. Kabir, A. M. R., Wada, S., Inoue, D., Tamura, Y., Kajihara, T., Mayama, H., Sada, K., Kakugo, A. \& Gong, J. P. Formation of ring-shaped assembly of microtubules with a narrow size distribution at an air-buffer interface. Soft Matter 8, 10863-10867 (2012).

35. Rothwell, S. W., Grasser, W. A. \& Murphy, D. B. End-to-end annealing of microtubules in vitro. J. Cell Biol. 102, 619-627 (1986). 
36. Prabhune, M., Von Roden, K., Rehfeldt, F. \& Schmidt, C. F. Sulfo-SMCC prevents annealing of taxol-stabilized microtubules in vitro. PLoS One 11, e0161623 (2016).

37. Inoue, D., Nitta, T., Kabir, A. M. R., Sada, K., Gong, J. P., Konagaya, A. \& Kakugo, A. Sensing surface mechanical deformation using active probes driven by motor proteins. Nat. Commun. 7, 12557 (2016).

38. Matsuda, K., Kabir, A. M. R., Akamatsu, N., Saito, A., Ishikawa, S., Matsuyama, T., Ditzer, O., Islam, M. S., Ohya, Y., Sada, K., Konagaya, A., Kuzuya, A. \& Kakugo, A. Artificial smooth muscle model composed of hierarchically ordered microtubule asters mediated by DNA origami nanostructures. Nano Lett. 19, 3933-3938 (2019). 


\section{Figure legends}

Fig. 1. Structures of (a) 5-chloro-6-[2,6-difluoro-4-[3-(methylamino)propoxy]phenyl]-N((2S)-1,1,1-trifluoropropan-2-yl)-[1,2,4]triazolo[1,5-a]pyrimidin-7-amine, ～i.e., cevipabulin, and (b) its derivative, (S)-6-(4-(3-butylamino(propoxy)-2,6difluorophenyl)-5-chloro-N-(1,1,1-trifluoro-propan-2-yl)-[1,2,4]triazolo[1,5-

a]pyrimidin-7-amine, i.e., cevipabulin-R, used in the study. The structure of the most common microtubule-stabilizing agent, (c) paclitaxel, is shown for comparison.

Fig. 2. Energetically most suitable complexes of $\beta$-tubulin and the cevipabulin molecule (i-iii) and of $\beta$-tubulin and the cevipabulin-R molecule (iv-vi) obtained from molecular docking using AutoDock Vina software. The results from the docking are summarized in Table 1 . The structures shown in (i) to (vi) correspond to modes $1,3,8,11,14$, and 18, respectively, which are marked by asterisks in Table 1 . The binding sites to which cevipabulin and cevipabulin-R are bound are indicated using red and green arrows, respectively.

Fig. 3. Fluorescence microscopy images of the stabilized microtubules in the presence of 0.1, 5.0, 50.0, and 100.0 $\mu \mathrm{M}$ (a) cevipabulin, (b) cevipabulin-R and (c) paclitaxel, with 
$5.60 \mu \mathrm{M}$ tubulin. The images were captured at $0 \mathrm{~h}$ of microtubule preparation. Scale bar: $10 \mu \mathrm{m}$.

Fig. 4. Effect of different microtubule-stabilizing agent concentrations on (a) mean lengths and (b) densities of the microtubules. Error bar= standard deviation. Microtubules observed just after preparation were analyzed, i.e., incubation time $=0 \mathrm{~h}$. In all cases, the concentration of tubulin was fixed at $5.60 \mu \mathrm{M}$.

Fig. 5. Persistence lengths of microtubules stabilized by cevipabulin, cevipabulin-R, or paclitaxel. The number of microtubules considered to determine the persistence lengths is mentioned in the legend. Values of $\mathrm{L}_{\mathrm{p}}$, the number of microtubules analyzed, $\mathrm{n}$ and the goodness of fit, $\mathrm{R}^{2}$, are mentioned in the figure legends. Solid lines are derived from the following equation:

$$
R^{2}=2 L_{p}^{2}\left[\frac{L}{L_{p}}-1+e^{\frac{L}{L_{p}}}\right]
$$

where $\mathrm{R}$ is the mean squared end-to-end length,

$\mathrm{L}$ is the contour length of a filament, and

$\mathrm{L}_{\mathrm{p}}$ derived from the fit represents the persistence length of the microtubule.

Fig. 6. Effect of incubation time on the mean lengths (a) and densities (b) of the microtubules stabilized by different microtubule-stabilizing agents. Error bar= standard deviation. Microtubules were incubated at room temperature and kept in the dark during 
incubation. The concentrations of tubulin and the microtubule-stabilizing drugs in all cases were $5.60 \mu \mathrm{M}$ and $50.0 \mu \mathrm{M}$, respectively. The mean lengths and numbers of microtubules analyzed are given in Table 2. 


\section{FIGURES}

(a)<smiles>CNCCCOc1cc(F)c(-c2c(Cl)nc3ncnn3c2NC(C)C(F)(F)F)c(F)c1</smiles>

Cevipabulin $\left(\mathrm{C}_{18} \mathrm{H}_{18} \mathrm{ClF}_{5} \mathrm{~N}_{6} \mathrm{O}\right)$ Molecular weight: 464.82 (b)<smiles>CCCCNCCCOc1cc(F)c(-c2c(Cl)nc3ncnn3c2NC(C)C(F)(F)F)c(F)c1</smiles>

Cevipabulin-R $\left(\mathrm{C}_{21} \mathrm{H}_{24} \mathrm{ClF}_{5} \mathrm{~N}_{6} \mathrm{O}\right)$ Molecular weight: 506.90

(c)<smiles>O=C(N[C@H](c1ccccc1)[C@H](O)C(=O)O)c1ccccc1</smiles>

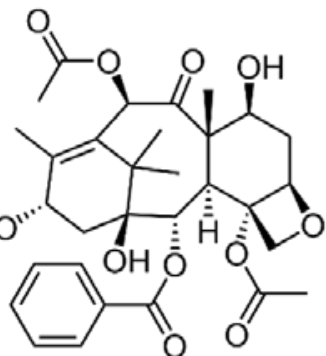

Paclitaxel $\left(\mathrm{C}_{47} \mathrm{H}_{51} \mathrm{NO}_{14}\right)$ Molecular weight: 853.91

Fig. 1. Structures of (a) 5-chloro-6-[2,6-difluoro-4-[3-(methylamino)propoxy]phenyl]-N((2S)-1,1,1-trifluoropropan-2-yl)-[1,2,4]triazolo[1,5-a]pyrimidin-7-amine, i.e., cevipabulin, and (b) its derivative, (S)-6-(4-(3-butylamino(propoxy)-2,6difluorophenyl)-5-chloro-N-(1,1,1-trifluoro-propan-2-yl)-[1,2,4]triazolo[1,5a]pyrimidin-7-amine, i.e., cevipabulin-R, used in the study. The structure of the most common microtubule-stabilizing agent, (c) paclitaxel, is shown for comparison. 
(a) Cevipabulin and $\beta$-tubulin complexes (i-iii)

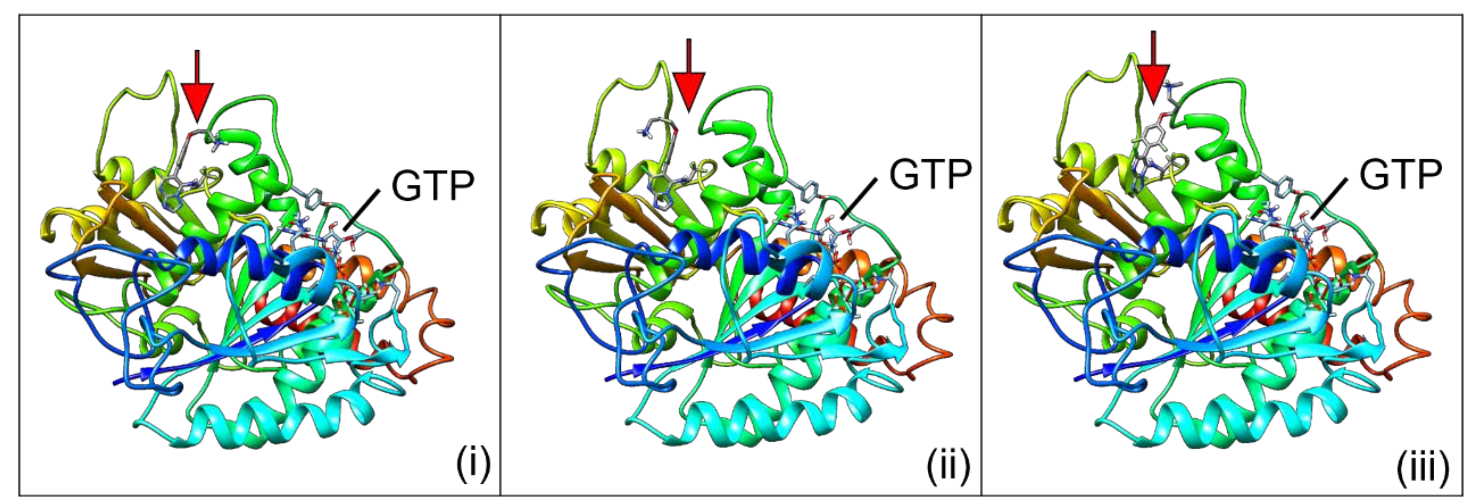

(b) Cevipabulin-R and $\beta$-tubulin complexes (iv-vi)

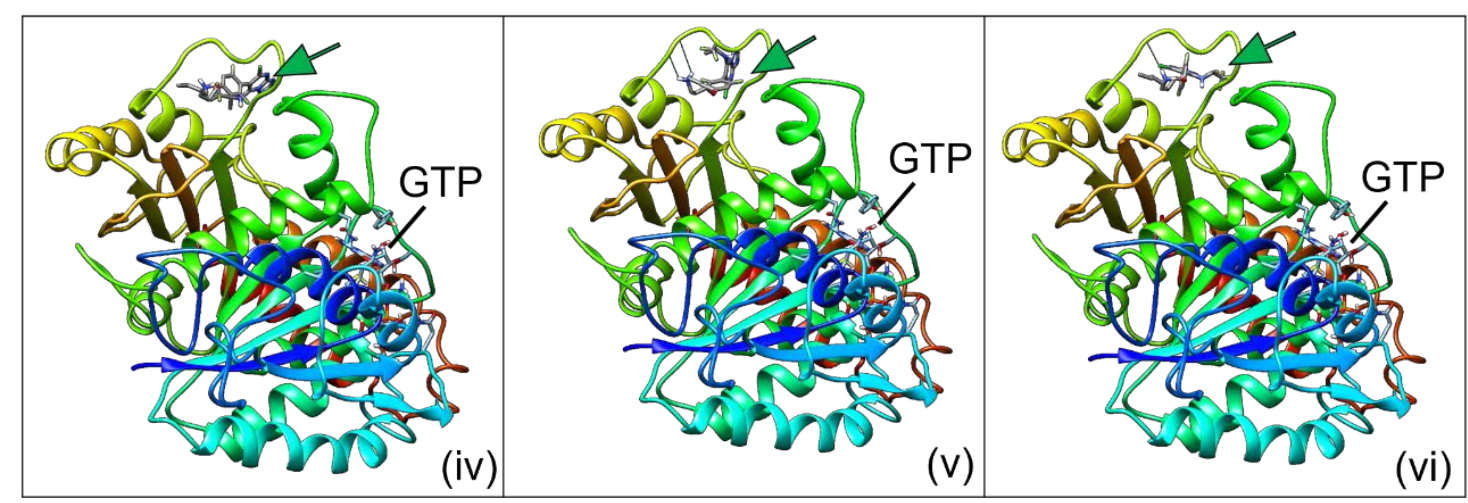

Fig. 2. Energetically most suitable complexes of $\beta$-tubulin and the cevipabulin molecule

(i-iii) and of $\beta$-tubulin and the cevipabulin-R molecule (iv-vi) obtained from molecular docking using AutoDock Vina software. The results from the docking are summarized in

Table 1. The structures shown in (i) to (vi) correspond to modes 1, 3, 8, 11, 14, and 18, respectively, which are marked by asterisks in Table 1 . The binding sites to which cevipabulin and cevipabulin- $\mathrm{R}$ are bound are indicated using red and green arrows, respectively. 
(a) Cevipabulin-stabilized microtubules
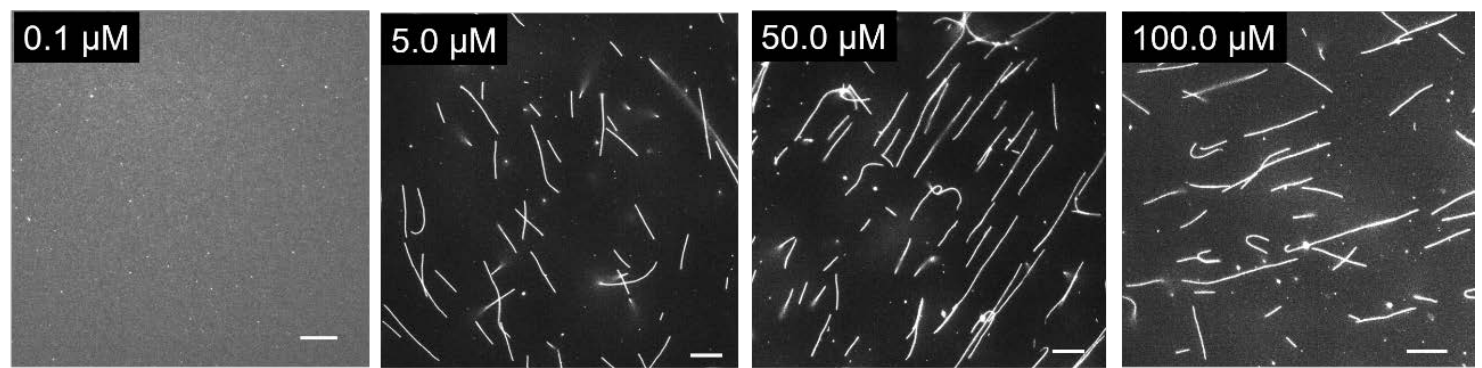

(b) Cevipabulin-R-stabilized microtubules
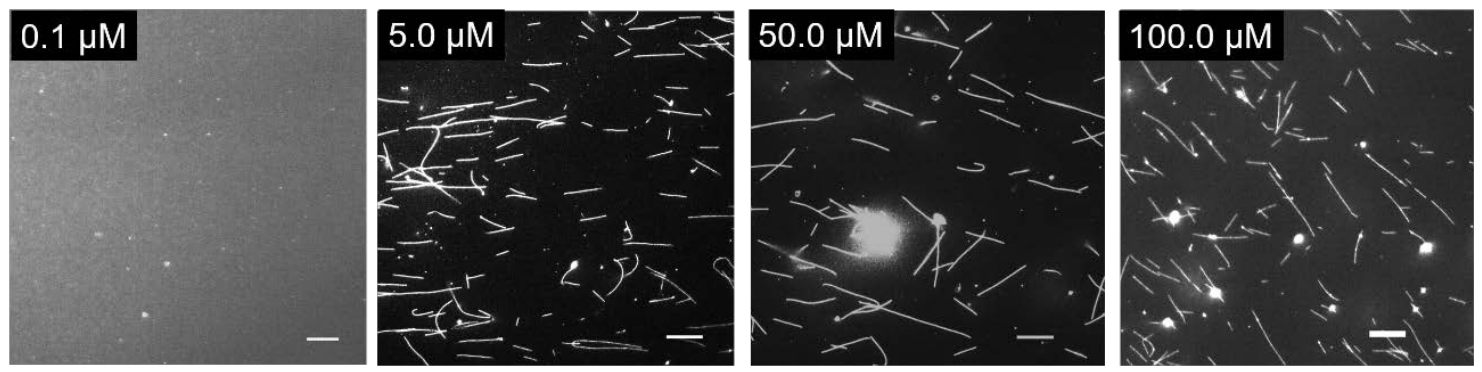

(c) Paclitaxel-stabilized microtubules
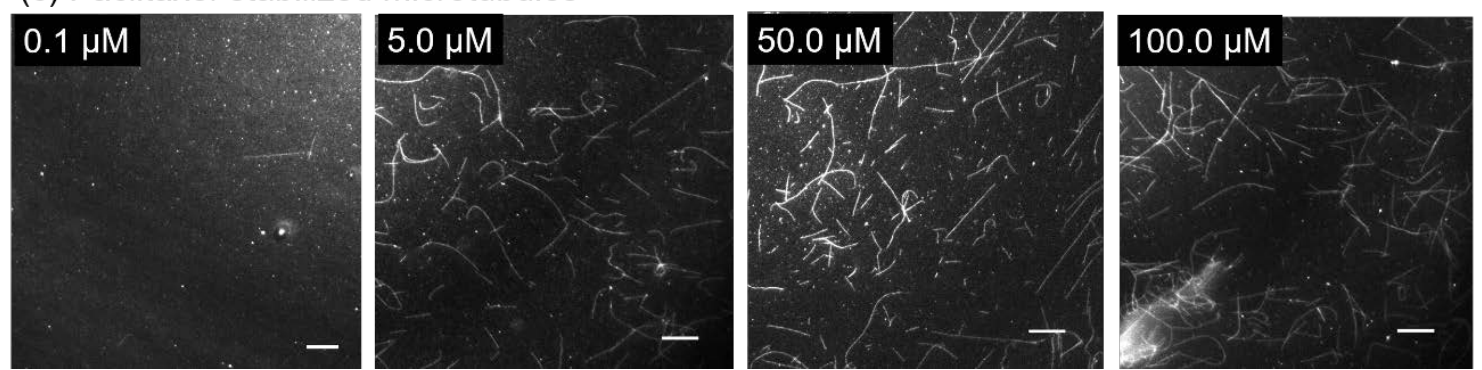

Fig. 3. Fluorescence microscopy images of the stabilized microtubules in the presence of

0.1, 5.0, 50.0, and 100.0 $\mu \mathrm{M}$ (a) cevipabulin, (b) cevipabulin-R and (c) paclitaxel, with

$5.60 \mu \mathrm{M}$ tubulin. The images were captured at $0 \mathrm{~h}$ of microtubule preparation. Scale bar:

$10 \mu \mathrm{m}$. 
(a)

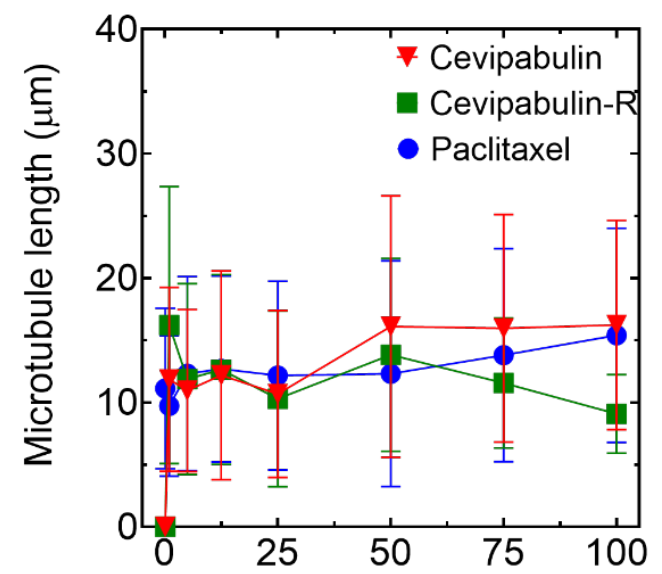

(b)

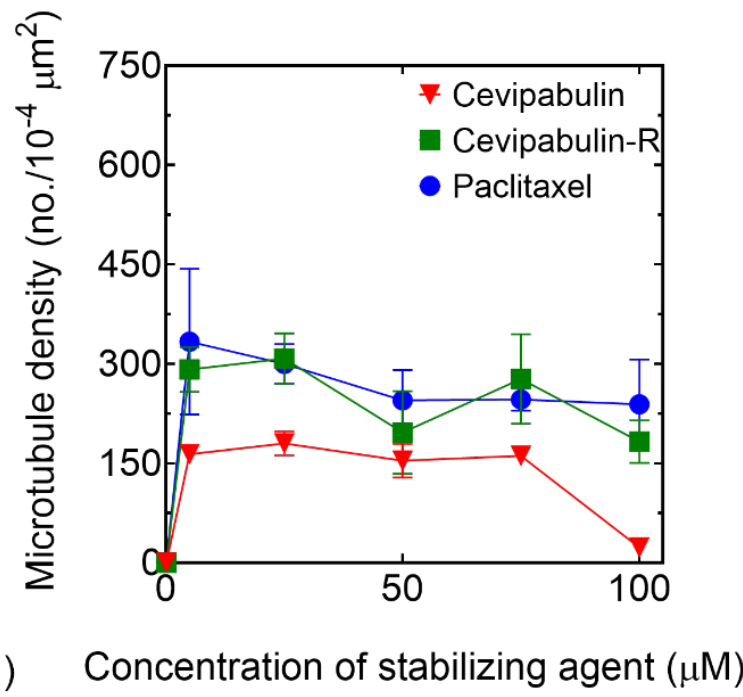

Fig. 4. Effect of different microtubule-stabilizing agent concentrations on (a) mean lengths and (b) densities of the microtubules. Error bar= standard deviation. Microtubules observed just after preparation were analyzed, i.e., incubation time $=0 \mathrm{~h}$. In all cases, the concentration of tubulin was fixed at $5.60 \mu \mathrm{M}$. 

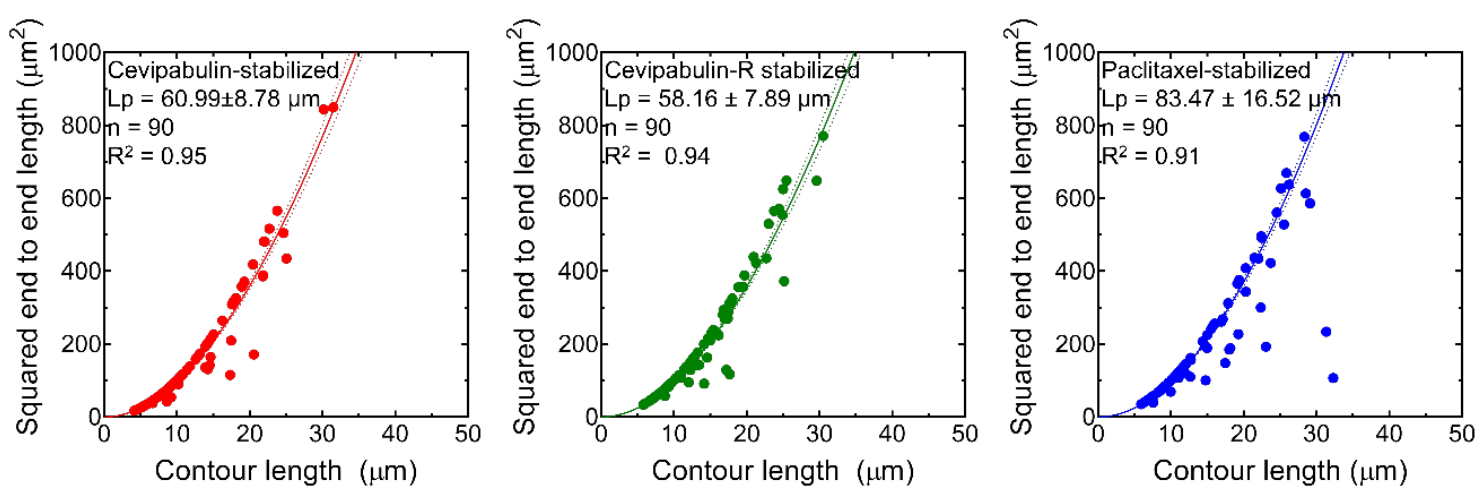

Fig. 5. Persistence lengths of microtubules stabilized by cevipabulin (left), cevipabulinR (middle), or paclitaxel (right). The number of microtubules considered to determine the persistence lengths is mentioned in the legend. Values of $L_{p}$, the number of microtubules analyzed, $\mathrm{n}$ and the goodness of fit, $\mathrm{R}^{2}$, are mentioned in the figure legends. Solid lines are derived from the following equation:

$$
R^{2}=2 L_{p}^{2}\left[\frac{L}{L_{p}}-1+e^{\frac{L}{L_{p}}}\right]
$$

where $\mathrm{R}$ is the mean squared end-to-end length,

$\mathrm{L}$ is the contour length of a filament, and

$\mathrm{L}_{p}$ derived from the fit represents the persistence length of the microtubule. 
(a)

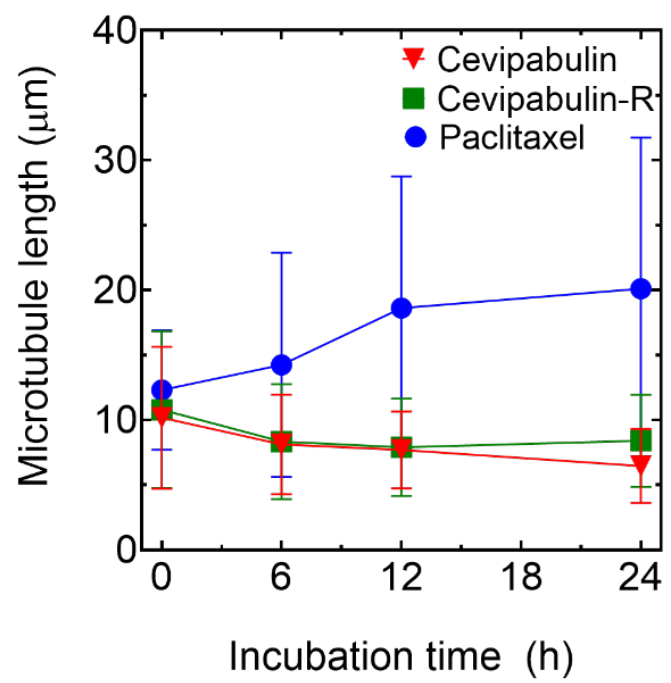

(b)

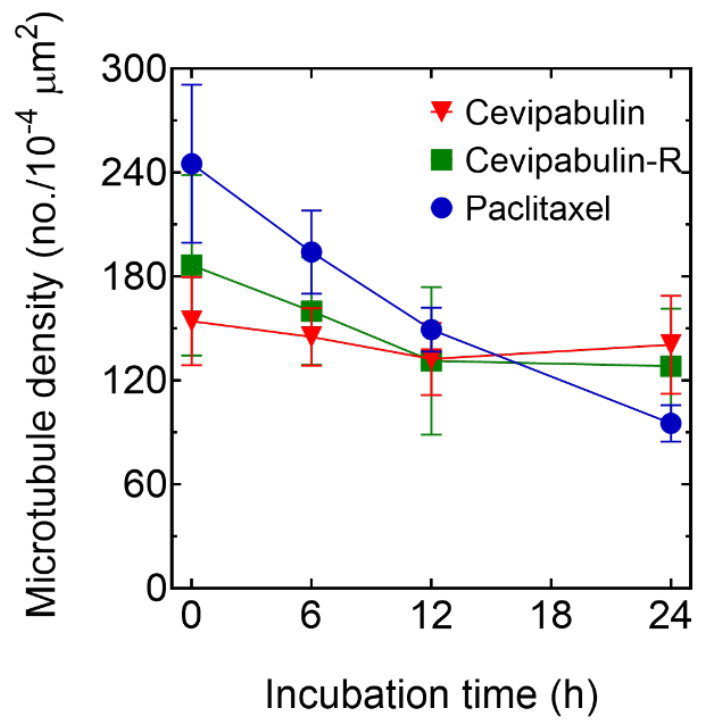

Fig. 6. Effect of incubation time on the mean lengths (a) and densities (b) of the microtubules stabilized by different microtubule-stabilizing agents. Error bar= standard deviation. Microtubules were incubated at room temperature and kept in the dark during incubation. The concentrations of tubulin and the microtubule-stabilizing drugs in all cases were $5.60 \mu \mathrm{M}$ and $50.0 \mu \mathrm{M}$, respectively. The mean lengths and numbers of microtubules analyzed are given in Table 2. 